Brief note

\title{
MODELLING OF RANDOM VERTICAL IRREGULARITIES OF RAILWAY TRACKS
}

\author{
M. PODWÓRNA \\ Department of Structural Dynamics \\ Wroclaw University of Technology \\ 27 Wyspiańskiego Street, PL-50370 Wroclaw, POLAND \\ E-mail: monika.podworna@pwr.edu.pl
}

\begin{abstract}
The study presents state-of-the-art in analytical and numerical modelling of random vertical irregularities of continuously welded ballasted railway tracks. The common model of railway track irregularity vertical profiles is applied, in the form of a stationary and ergodic Gaussian process in space. Random samples of track irregularity vertical profiles are generated with the Monte-Carlo method. Based on the numerical method developed in the study, the minimum and recommended sampling number required in the random analysis of railway bridges and number of frequency increments (harmonic components) in track irregularity vertical profiles simulation are determined. The lower and upper limits of wavelengths are determined based on the literature studies. The approach yields track irregularity random samples close to reality. The track irregularity model developed in the study can be used in the dynamic analysis of railway bridge / track structure / highspeed train systems.
\end{abstract}

Key words: ballasted railway track, continuously welded track, random vertical irregularities, track irregularities modeling, track irregularities random samples, Monte-Carlo method.

\section{Introduction}

Railway bridges loaded by high-speed trains need to be designed or modernized to ensure the traffic safety condition and the passenger comfort condition. Too large vertical accelerations of the bridge platform may cause ballast destabilization in the track structure (ERRI, 1999). Railway track irregularities are commonly considered to be one of the main factors affecting the dynamic response of bridge / track structure / high-speed train systems (BTT). So far, theoretical studies on the effect of track irregularities on vibrations of railway bridges loaded by high-speed trains were conducted on one-dimensional / three-dimensional (1D/3D) simplified models of a BTT system.

$\mathrm{Au}$ et al. (2002) developed a 1D vibration study on railway cable-stayed bridges under moving trains, taking into account random rail irregularities. The main girder of the bridge is modelled using 6DOF Euler beam finite elements and taking into account the linear and geometric stiffness matrices. Double-side constraints between the moving wheel set unsprung masses and the rails are assumed. The track structure is neglected. The matrix equation of motion of the bridge / moving train system is formulated in the explicit form. Sample vertical profiles of random rail roughness, considered as stationary and ergodic processes in space, are generated using the empirical formula for PSD function with the parameters corresponding to the USA railway classes $1-6$.

Lu et al. (2009) adopted the vehicle - bridge interaction (VBI) element in non-stationary random vibration analysis of vehicle / bridge systems. The VBI element condenses DOFs of the vehicle into those of the bridge by using the Newmark integration scheme. The bridge is reflected by a prismatic beam discretized with Euler beam finite elements. The train is composed of a number of vehicles, each reflected by the 6DOF Matsuura four-axle model. 
The dynamic response of an existing bridge subjected to different moving trains is developed by Majka and Hartnett (2009), including track irregularities and neglecting snaking of wheel sets. Railway track vertical irregularities are described by a stationary and ergodic Gaussian process in space. The research was carried out using the 3D dynamic bridge - train interaction (DBTI) model, in which the inertia forces of the moving unsprung train axles are coupled with the bridge.

The study presents state-of-the-art in analytical and numerical modelling of random vertical irregularities of continuously welded ballasted railway tracks. The common model of railway track irregularity vertical profiles is applied in the form of a stationary and ergodic Gaussian process in space. Random samples of track irregularity vertical profiles are generated with the Monte-Carlo method. The numerical parameters of the Monte-Carlo simulation are determined based on the method developed in the study.

\section{Modelling of random vertical irregularities of continuously welded ballasted railway tracks}

Railway track irregularities are due to track formation technology, wear, clearances, ground subsidence, contemporary mechanical maintenance, settlement and other factors. In this study, modelling of random vertical irregularities of railway tracks is addressed to bridge / track structure / high-speed train systems. Therefore, the following assumptions are adopted:

- The track axis is a horizontal straight line.

- The ballasted track structure has continuously welded rails.

- There are random vertical track irregularities (elevation irregularities) resulting from the construction and maintenance of the track, as well as from the settlement of ballast and subgrade.

- Short wavelength corrugation irregularities in rail and design geometry irregularities in track formation are not taken into consideration.

- Random vertical track irregularities are identical for both operating rails.

Experimental measurements and/or modelling of track irregularities are considered in a number of References; the most important of them are discussed below. Esveld (1986) introduced a classification of track irregularities as shown in Tab.1. Under the adopted assumptions, vertical track irregularities with wavelengths of $0.10-70.00 \mathrm{~m}$ are taken into consideration, which produce vertical vibrations in bridge / track structure / moving train systems at frequencies of $\sim 1.1-800 \mathrm{~Hz}$ for trains operating at speeds up to $300 \mathrm{~km} / \mathrm{h}$.

Fryba (1996) states that railroad track irregularities are an important source of vibration excitation of bridges during passages of high-speed trains. The researcher distinguishes four types of track irregularities:

- average vertical profile of two rails,

- average horizontal profile of two rails,

- difference in vertical rail profiles,

- difference in horizontal rail profiles, measured $14 \mathrm{~mm}$ below the running surface of the rail.

Railway track irregularities are limited by railway administrations in different countries, depending on operating speeds. 
Table 1. Classification of track irregularities proposed by Esveld (1986).

\begin{tabular}{cc}
\hline wavelength $L_{r}[\mathrm{~m}]$ & type of irregularities in rail \\
\hline $0.03-0.10$ & short wavelength corrugation \\
$0.10-1.00$ & long wavelength corrugation \\
$1.00-3.00$ & long waves and rolling defects \\
\hline & \\
\hline wavelength $L_{r}[\mathrm{~m}]$ & type of irregularities in formation \\
\hline $3.00-25.00$ & alignment, cant, twist, gauge etc. \\
$25.00-70.00$ & alignment \\
$>70.00$ & design geometry \\
\hline
\end{tabular}

The most common model of railway track irregularities in the vertical profile is adopted as a stationary ergodic Gaussian process in space, described by a random function $r(x)$ of zero expectance and constant variance. All statistical characteristics of $r(x)$, calculated with one sample of this process are equal to the characteristics of a set of function tests. Track irregularities in the vertical profile are characterized by one-sided power spectral density function (PSD) $S_{r r}(\Omega)$ where $\Omega=2 \pi / L_{r}[\mathrm{rad} / \mathrm{m}]$ is spatial frequency or wave number. In the literature, a number of analytical expressions for the PSD function is presented. For example, Zhang et al. (2001) show the PSD function, previously used by Wiriyachai et al. (1982), in the form

$$
S_{r r}(\Omega)=A \frac{\Omega_{c}^{2}}{\left(\Omega^{2}+\Omega_{c}^{2}\right) \Omega^{2}} \quad\left[m m^{2} m / r a d\right]
$$

where

$$
\begin{aligned}
& A=3.365\left[\mathrm{~mm}^{2} \mathrm{rad} / \mathrm{m}\right]-\text { vertical track irregularity parameter, } \\
& \Omega_{c}=0.8242[\mathrm{rad} / \mathrm{m}]-\text { critical number. }
\end{aligned}
$$

Random samples to the track irregularity function in the vertical plane are determined from the inverse Fourier transform (Zhang et al., 2001), i.e.

$$
r(x)=2 \sum_{n=1}^{N} \sqrt{S_{r r}\left(\Omega_{n}\right) \Delta \Omega} \cos \left(\Omega_{n} x+\phi_{n}\right)
$$

where

$\left.\Omega_{n}=\Omega_{\min }+(n-0.5)\right) \Delta \Omega-$ discrete frequency of the PSD function,

$\phi_{n}$ - random phase angle uniformly distributed over $[0.2 \pi][\mathrm{rad}]$ interval and independent of $n=1,2, \ldots, N$, $\Delta \Omega=\left(\Omega_{\max }-\Omega_{\min }\right) / N-$ frequency increment,

$N$ - total number of frequency increments in $\left[\Omega_{\min }, \Omega_{\max }\right]$ interval,

$\Omega_{\min }=2 \pi / L_{r, \max }, \Omega_{\max }=2 \pi / L_{r, \text { min }}$ - lower and upper limits of spatial frequency defining range in which PSD function is included,

$L_{r, \min }, L_{r, \max }-$ lower and upper limits of wavelength defining range in which the PSD function is included.

The value of $N$ should be properly large. Zhang et al. (2001) assumed $N=500$. Random sampling according to Eq.(2.2) corresponds to the Monte-Carlo method.

Lei and Noda (2002) adapted a similar formula as in Eq. (2.2) for $r(x)$, but instead of cosine they used a sine function and interpreted the expression $\sigma_{n}=2 \sqrt{S_{r r}\left(\Omega_{n}\right) \Delta \Omega}$ as the standard deviation $a_{n}$ of Gaussian 
random variable with zero expectance and independent of $n=1,2, \ldots, N$. Furthermore, $a_{n}$ and $\phi_{n}$ are independent of each other. The PSD function of the random variable $r(x)$ corresponds to line grades 1 to 6 according to the American Railway Standard developed by the US Federal Railroad Administration (FRA), and has the form

$$
S_{r r}(\Omega)=k A \frac{\Omega_{c}^{2}}{\left(\Omega^{2}+\Omega_{c}^{2}\right) \Omega^{2}} \quad\left[\frac{m m^{2} m}{r a d}\right]
$$

where $k=0.25, \Omega_{c}=0.8245[\mathrm{rad} / \mathrm{m}]$. The values of coefficient $A$ are given in Tab.2.

Line grades from 1 to 6 are classes of quality of railway lines. Grade 1 is the worst, unacceptable on high-speed lines, whereas grade 6 is the best. Lei and Noda (2002) considered all six grades and assumed $L_{r, \min }=0.50 \mathrm{~m}, L_{r, \max }=50.00 \mathrm{~m}$.

Table 2. Values of coefficient $A$ according to the American Railway Standard (Lei and Noda, 2002).

\begin{tabular}{cc}
\hline Line grade & $A\left[\mathrm{~mm}^{2} \mathrm{rad} / \mathrm{m}\right]$ \\
\hline 1 & 121.07 \\
2 & 101.81 \\
3 & 68.16 \\
4 & 53.76 \\
5 & 20.95 \\
6 & 3.39 \\
\hline
\end{tabular}

Au et al. (2002) generated vertical profiles of random irregularities of a railway track using the PSD function in the form of

$$
S_{r r}(\Omega)=A \frac{\Omega_{2}^{2}\left(\Omega^{2}+\Omega_{l}^{2}\right)}{\left(\Omega^{2}+\Omega_{2}^{2}\right) \Omega^{4}} \quad\left[\frac{m^{3}}{r a d}\right]
$$

where, for class 4 , they assumed

$$
A=2.75 \cdot 10^{-8}\left[\mathrm{~m}^{2} \mathrm{rad} / \mathrm{m}\right], \Omega_{1}=0.0233, \Omega_{2}=0.131[\mathrm{rad} / \mathrm{m}]
$$

In the literature, one can find examples of railway track random irregularities in accordance with Eq.(2.2), for $N=2000, L_{r, \min }=1.00 m, L_{r, \max }=628 \mathrm{~m}$. Random samples for other line grades of tracks were generated proportionally. The initial random vertical motion of rail vehicles prior to entering the bridge was induced by the access zone with a length of $200 \mathrm{~m}$.

Au et al. (2002) calculated the basic statistics (mean value and standard deviation) for each set of results obtained for a selected operating speed of the train. The researchers stated that the distribution is normal if the results differ to $16 \%$ of the expected value plus the standard deviation. On the basis of numerical analysis of the bridge / railway track, the writers argue that the number of samples $N_{s}=20$ is sufficient.

Song et al. (2003) applied the PSD function described by Eq.(2.4) for track irregularities with $N=1024, L_{r, \min }=1.524 \mathrm{~m}, L_{r, \max }=304.8 \mathrm{~m}$. Lu et al. (2009) also considered a stationary spatial random process $r(x)$ corresponding to railway track roughness. They used the American PSD function describing the irregularities to line grade 6, according to Eq.(2.3) and Tab.2, using the following values of numerical simulation parameters: $N=100, L_{r, \min }=1.39 \mathrm{~m}, L_{r, \max }=\infty, N_{s}=500$. The PSD function according to 
Eq.(2.3) and Tab.2 is adopted by Zhang et al. (2001) but for $L_{r}>1.00 \mathrm{~m}$ and line grades 4-6. The writers tested the samples obtained by the Monte Carlo method and believe that $N_{s}=100$ is too small for random analysis of track/train systems.

\section{Numerical analysis}

The objectives of the numerical analysis are as follows:

- determination of the minimum and recommended number $N$ of harmonics of the ergodic stationary process modelling vertical profiles of track irregularities close to reality,

- determination of the minimum and recommended sampling number $N_{s}$ required in the random analysis of BTT systems.

The numerical method developed in the study consists of the following steps:

1) statistical analysis of the random variable $\bar{r}=\max |r(x)|[\mathrm{mm}], x \in[0 ; 100][\mathrm{m}]$ as a function of the parameters $N, N_{s}$,

2) determination of the minimum and recommended sampling number $N_{s}$ to determine the basic statistics, i.e., the expectance $E[\bar{r}]$ and standard deviation $D[\bar{r}]$,

3) determination of the minimum and recommended number of harmonics $N$ to calculate the above statistics so as to be independent of the value of $N$.

Taking into account the discussion presented in Section 2, vertical track irregularities with wavelengths of $0.10-70.00 \mathrm{~m}$ are considered.

On the basis of Eqs (2.2)-(2.3) and Tab.2, the authors developed a computer programme in Delphi to generate random samples $r(x)$ and to calculate statistics $E[\bar{r}], D[\bar{r}]$. Table 3 shows the statistics of a random variable $\bar{r}[\mathrm{~mm}]$ as a function of the sampling number $N_{s}$, for line grades $L_{g}=4,5,6$. The values correspond to the recommended value of $N=2000$, determined in further considerations. The calculations were performed for line grade $L_{g}=4$, while the statistics corresponding to $L_{g}=5$ and 6 were calculated using the following formulae resulting from Eqs (2.2)-(2.3)

$$
\left.E[\bar{r}]\right|_{L_{g}=i}=\left.\beta_{i} E[\bar{r}]\right|_{L_{g}=4},\left.\quad D[\bar{r}]\right|_{L_{g}=i}=\left.\beta_{i} D[\bar{r}]\right|_{L_{g}=4}, i=5,6
$$

where

$$
\beta_{i}=\sqrt{A\left[L_{g}=i\right] / A\left[L_{g}=4\right]}
$$

Table 3. The basic statistics of the random variable $\bar{r}[\mathrm{~mm}]$ as a function of the sampling number $N_{s}$ for line grades $L_{g}=4,5,6$ and $N=2000$.

\begin{tabular}{ccccccc}
\hline & \multicolumn{2}{c}{$L_{g}=4$} & \multicolumn{2}{c}{$L_{g}=5$} & \multicolumn{2}{c}{$L_{g}=6$} \\
\cline { 2 - 7 }$N_{s}$ & $\begin{array}{r}E[\bar{r}] \\
(\mathrm{mm})\end{array}$ & $\begin{array}{c}D[\bar{r}] \\
(\mathrm{mm})\end{array}$ & $\begin{array}{c}E[\bar{r}] \\
(\mathrm{mm})\end{array}$ & $\begin{array}{c}D[\bar{r}] \\
(\mathrm{mm})\end{array}$ & $\begin{array}{c}E[\bar{r}] \\
(\mathrm{mm})\end{array}$ & $\begin{array}{c}D[\bar{r}] \\
(\mathrm{mm})\end{array}$ \\
\hline 100 & 37.4 & 6.7 & 23.4 & 4.2 & 9.4 & 1.7 \\
200 & 37.3 & 6.6 & 23.3 & 4.1 & 9.4 & 1.7 \\
300 & 37.4 & 6.8 & 23.3 & 4.2 & 9.4 & 1.7 \\
\hline
\end{tabular}




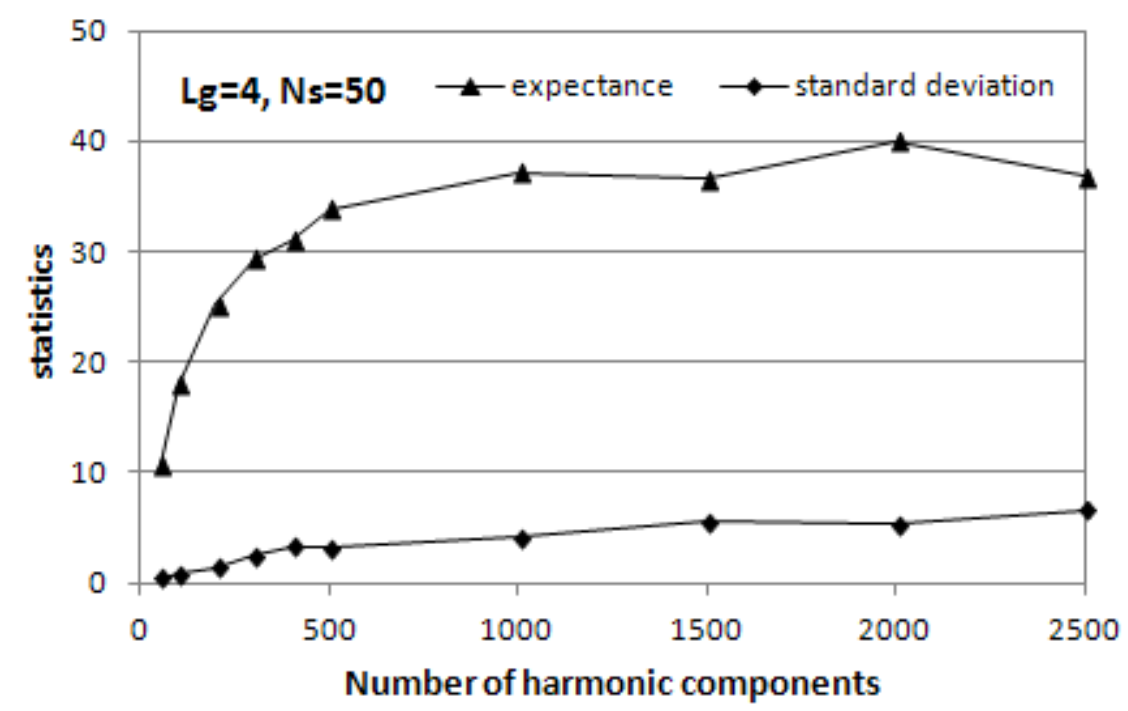

Fig.1. The basic statistics of the random variable $\bar{r}$ for $L_{g}=4$ and $N_{s}=50$.

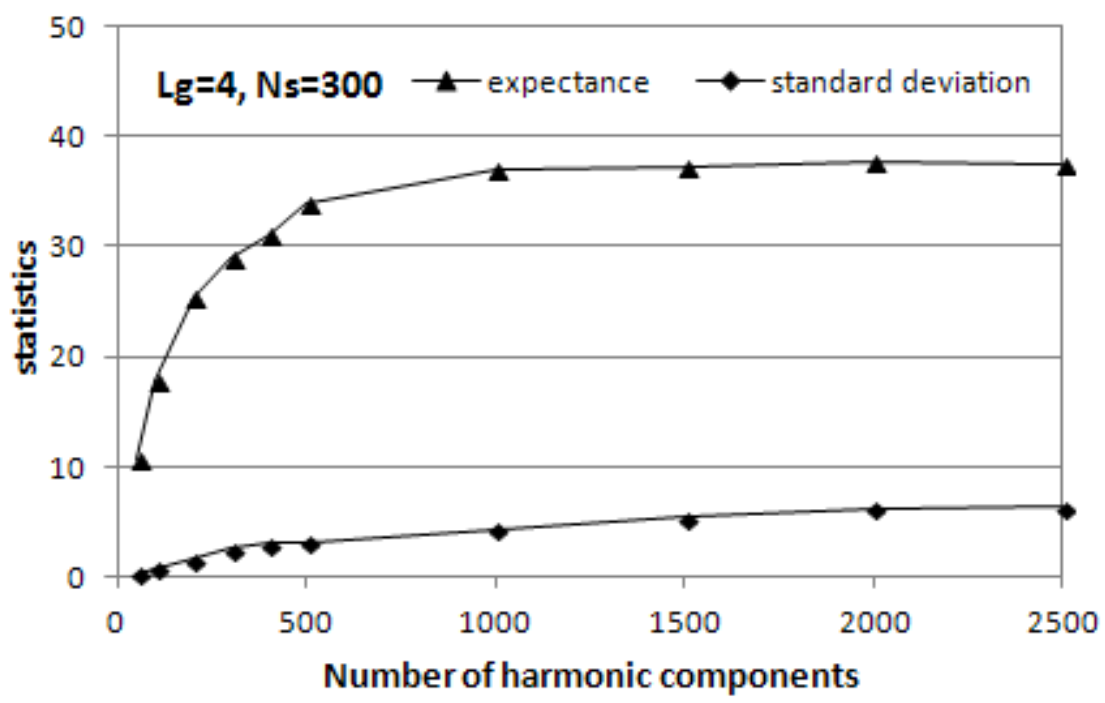

Fig.2. The basic statistics of the random variable $\bar{r}$ for $L_{g}=4$ and $N_{s}=300$.

Figure 1 presents the basic statistics of the random variable $\bar{r}$ for $L_{g}=4$ and $N_{s}=50$, as a function of a number of harmonic components $N$. Analogous results for $L_{g}=4$ and $N_{s}=300$ are presented in Fig.2. The authors also analyzed the results corresponding to $50<N_{s}<300$. On the basis of the graphs as shown in Figs 1 and 2, the recommended values of $N=2000, N_{s}=300$, and acceptable values of $N=1500, N_{s}=100$ are proposed.

Figures 3 and 4 show random samples of vertical track irregularity profiles $r(x)[\mathrm{mm}]$ for $L_{g}=4, N=1000$ and $N=2000$, respectively. The simulated profiles confirm the conclusion that $N=2000$ yields profiles close to reality. 


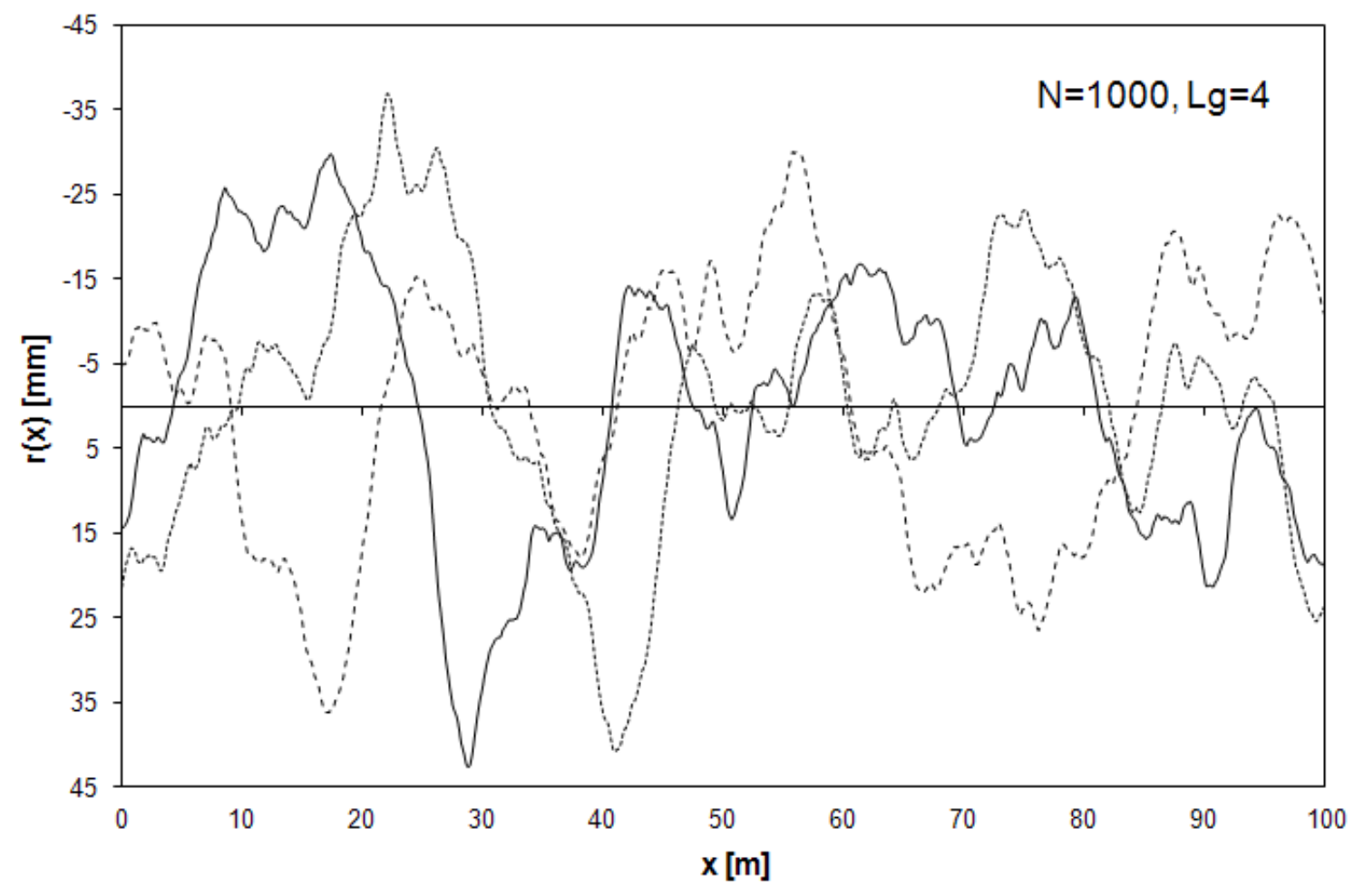

Fig.3. The random sample of vertical track irregularity profiles $r(x)[\mathrm{mm}]$ for $L_{g}=4, N=1000$.

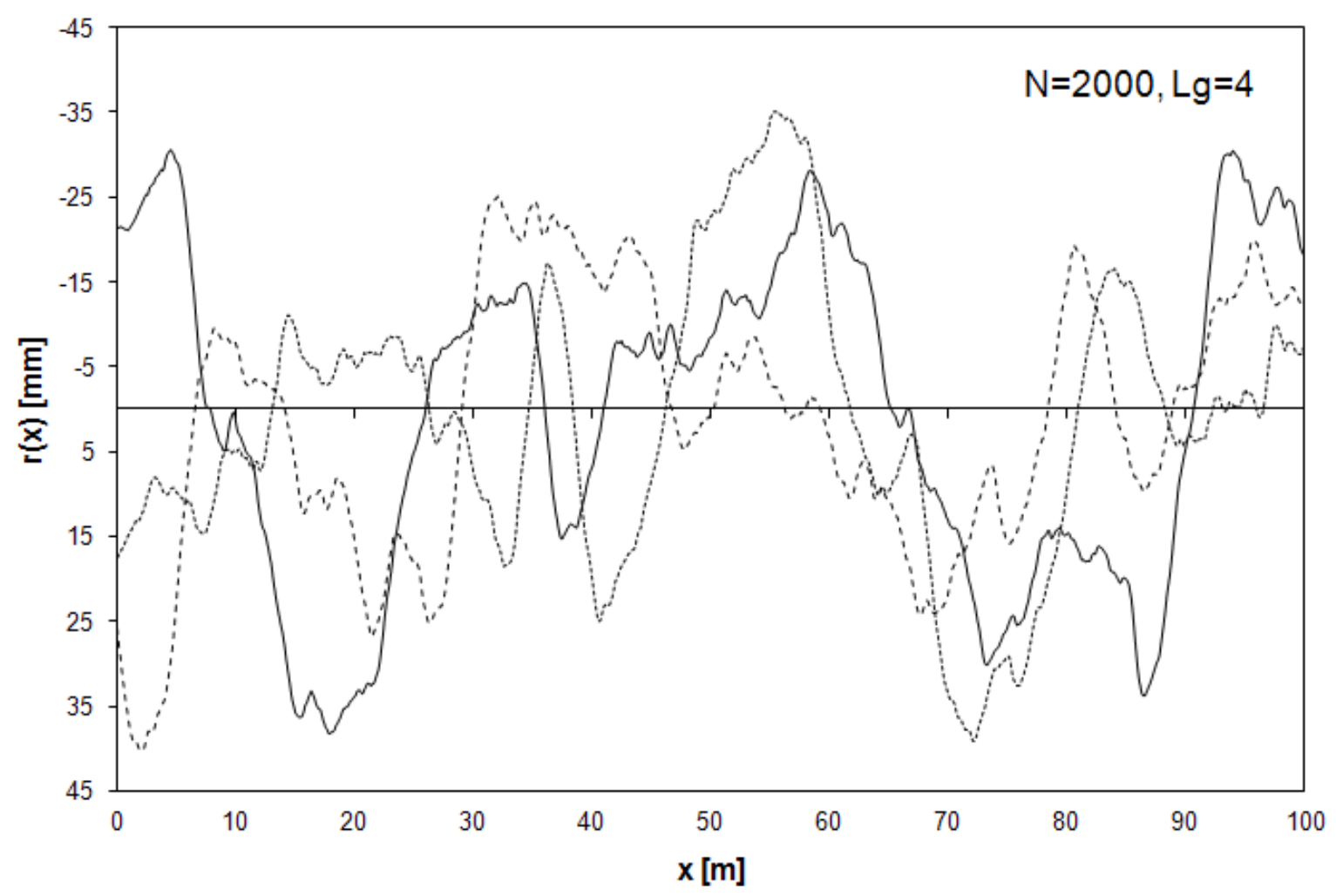

Fig.4. The random sample of vertical track irregularity profiles $r(x)[\mathrm{mm}]$ for $L_{g}=4, N=2000$. 


\section{Conclusions}

The track irregularity vertical profiles model developed in the study can be used in the random dynamic analysis of BTT systems.

In the study, the recommended and acceptable values of numerical parameters $N$ and $N_{s}$ are determined, for use in simulation of random processes in BTT dynamic systems, taking into account vertical random track irregularity. The parameter $N$ is the number of harmonics of a stationary ergodic random process describing vertical irregularities of a continuously welded ballasted railway track, whereas the parameter $N_{s}$ is the number of random samples in the dynamic analysis of BTT systems. The recommended values are equal to $N=2000, N_{s}=300$, and the acceptable values amount to $N=1500, N_{s}=100$.

The recommended and acceptable values of the $N$ and $N_{s}$ parameters have been determined using the numerical method developed in the study. The recommended values result in track irregularity random vertical profiles very close to reality. The acceptable values give acceptable approximate results.

\section{Acknowledgements}

The study was supported by the National Centre for Science, Poland, as a part of the project No. N N506 0992 40, realized in the period 2011-2013. This support is gratefully acknowledged.

\section{Nomenclature}

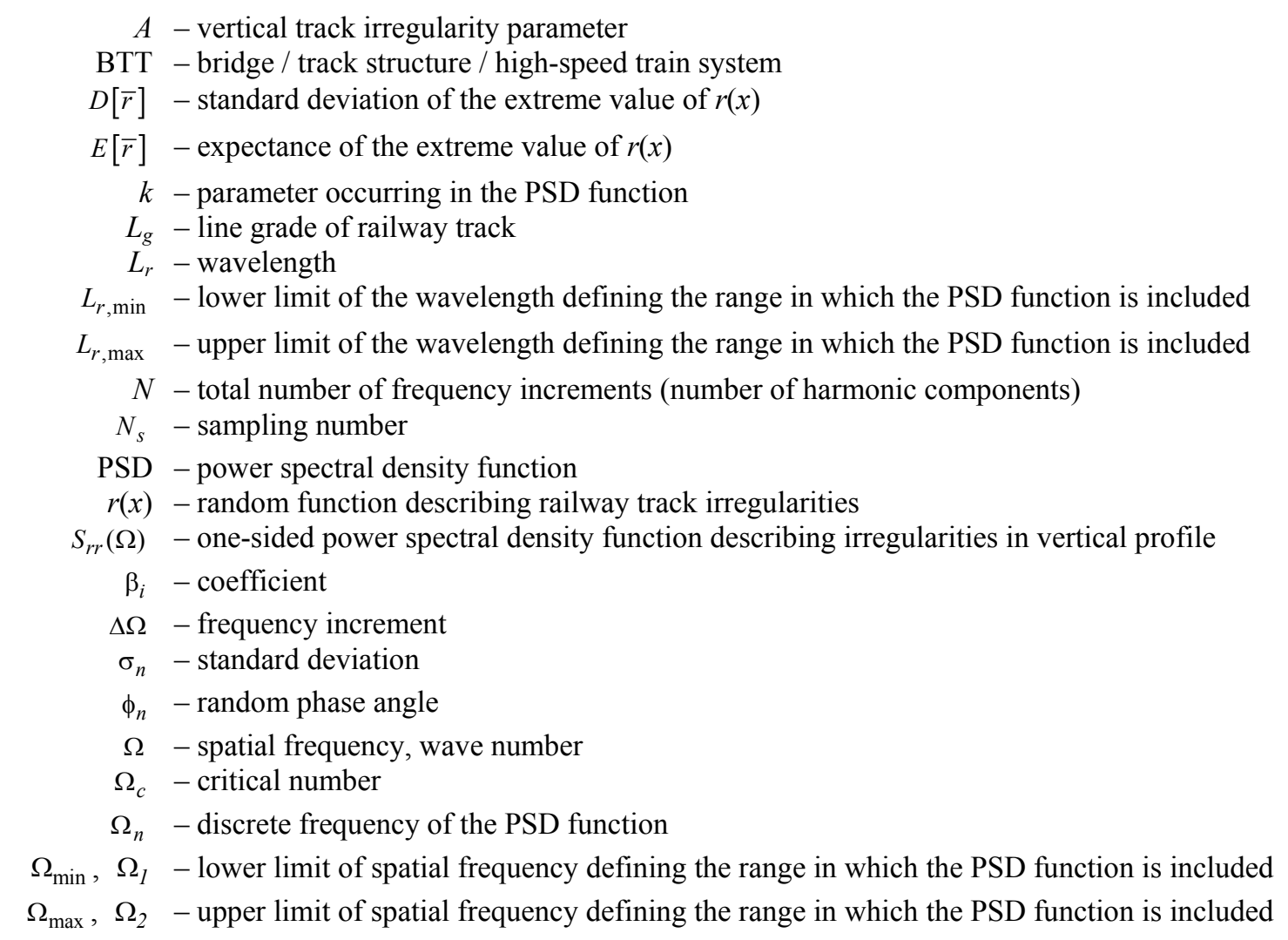

\section{References}

Au F.T.K., Wang J.J. and Cheung Y.K. (2002), Impact study of cable stayed railway bridges with random rail irregularities. - Engng. Struct., vol.24, pp.529-541. 
ERRI (1999). D214/RP9, Part B, Rail bridges for speeds $>200 \mathrm{~km} / \mathrm{h}$, Research Report, European Rail Research Institute, Utrecht, The Netherlands.

Esveld C. (1986): Measuring and rectifying rail roughness and bad welds. $-3^{\text {rd }}$ Int. Heavy Haul Railways Conf., Paper BIB-52, Vancouver, Canada.

Fryba L. (1996): Dynamics of railway bridges. - Academia, Praha, Czech Republic.

Lei X. and Noda N.A. (2002): Analyses of dynamic response of vehicle and track coupling system with random irregularity of track vertical profile. - J. Sound Vib., vol.258, pp.147-165.

Lu F., Lin J.H., Kennedy D. and Williams F.W. (2009): An algorithm to study non-stationary random vibrations of vehicle - bridge system. - Comp. and Struct., vol.87, pp.177-185.

Majka M. and Hartnett M. (2009): Dynamic response of bridges to moving trains: a study on effects of random track irregularities and bridge skewness. - Comput. Struct., vol.87, pp.1233-1253.

Podworna M. and Klasztorny M. (2014): Vertical vibrations of composite bridge / track structure / high-speed train system. Part 2: Physical and mathematical modelling. - Bul. Pol. Ac. 62, pp.181-196.

Song M.-K., Noh H.-C. and Choi C.-K. (2003): A new three dimensional finite element analysis model of high-speed train - bridge interactions. - Engng. Struct., vol.25, pp.1611-1626.

Wiriyachai A., Chu K.H. and Gang V.K. (1982): Bridge impact due to wheel and track irregularities. - ASCE J. Engng. Mech. Div., vol.108, pp.648-666.

Zhang Y.-W., Lin J.-H., Zhao Y., Howson D.P. and Williams F.W. (2010): Symplectic random vibration analysis of a vehicle moving on an infinitely long periodic track. - J. Sound and Vib., vol.329, pp.4440-4454.

Zhang Q.-L., Vrouwenvelder A. and Wardenier J. (2001): Numerical simulation of train - bridge interactive dynamics. - Comp. and Struct., vol.79, pp.1059-1075. 